\title{
Chronic stress, physiological adaptation and developmental programming of the neuroendocrine stress system
}

\author{
James A Coffman*,1 \\ ${ }^{1} \mathrm{MDI}$ Biological Laboratory, Kathryn W Davis Center for Regenerative Biology and Aging, Salisbury Cove, ME 04672, USA \\ *Author for correspondence: jcoffman@mdibl.org
}

Chronic stress undermines physical and mental health, in part via dysregulation of the neuroendocrine stress system. Key to understand this dysregulation is recognizing that the problem is not stress per se, but rather its chronicity. The optimally functioning stress system is highly dynamic, and negative feedback regulation enforces transient responses to acute stressors. Chronic stress overrides this, and adaptation to the chronicity can result in persistent dysregulation by altering sensitivity thresholds critical for control of system dynamics. Such adaptation involves plasticity within the central nervous system (CNS) as well as epigenetic regulation. When it occurs during development, it can have persistent effects on neuroendocrine regulation. Understanding how chronic stress programs development of the neuroendocrine stress system requires elucidation of stress-responsive gene regulatory networks that control CNS plasticity and development.

First draft submitted: 26 June 2019; Accepted for publication: 17 September 2019; Published online: 14 January 2020

Keywords: adaptation $\bullet$ chronic stress $\bullet$ cortisol $\bullet$ development $\bullet$ epigenetic $\bullet$ feedforward $\bullet$ glucocorticoid receptor - HPA axis • mineralocorticoid receptor $\bullet$ negative feedback

It is widely appreciated that chronic stress undermines health and accelerates degenerative aging, but how it does is a complex problem that calls for interdisciplinary research with a developmental perspective [1-3]. There is consensus that a key aspect of the problem involves dysregulation of the neuroendocrine system that orchestrates the physiological adjustments needed for homeostasis in a constantly changing and at times stressful environment. Such dysregulation is itself a source of chronic stress, compounding the problem. The fact that chronic stress is both a cause and effect of neuroendocrine dysregulation points up a key insight that is not as widely appreciated: stress per se is not a bad thing - in fact, it is essential for life, motivating core processes and behaviors that maintain health and homeostasis. Stress only becomes unhealthy ('toxic stress') when it exceeds the environmental resources available for its mitigation and the body's inherent capacity to adapt and thrive. Thus, the toxicity of chronic stress stems not so much from the stress per se, but from its chronicity. This essay asks the question of why that is the case and proposes the answer that the toxicity of chronic stress is a side effect of adaptation to the chronicity in developmentally plastic systems.

A defining attribute of living systems is that they are adaptive. Adaptation occurs at many levels of organization, from the biochemical up through individuals, populations and species. In general, biological systems adapt to changing conditions by changing their physiology, anatomy and behavior to meet the existential demands that those conditions impose, which can lead to the system acquiring new traits and abilities. At the level of a species this occurs through natural selection working on genetic variation in the population, facilitated to varying degrees by environmentally responsive developmental plasticity and epigenetic memory [4-7]. While species-level adaptations are typically construed as anatomical or physiological characteristics (such as wings, lungs, horns, etc.), some of the most powerful adaptations are behavioral characteristics such as sociality. Sociality is the adaptation that allowed the human species to organize to become the dominant force for environmental change that we now are. It is no accident then that social networks and associated safety nets are among the most effective environmental resources we have available for stress mitigation [8,9]. Conversely, negative social interactions or status can be a significant source of chronic stress $[10,11]$.

Future Medicine 
(A)

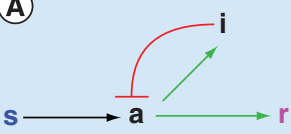

(B)

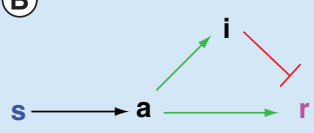

(C)

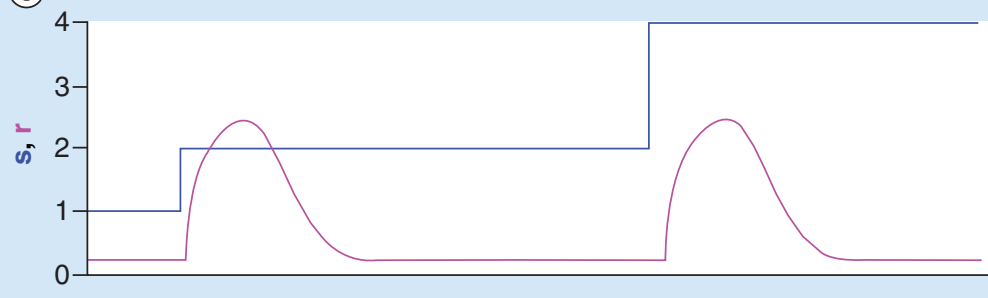

Time

Figure 1. Regulatory circuits for physiological adaptation. Adaptation to a chronic stimulus (s) can occur through either (A) negative feedback regulation of the activator (a), or (B) incoherent feedforward regulation of the response (r), by an inhibitor (i). This manifests temporally as (C) a transient increase in response in to a chronic increase in stimulus. Incoherent feedforward regulation also affords fold-change detection, which means that a subsequent response of the same amplitude will occur only when the fold-change of a subsequent increase in stimulus strength is equivalent to that which elicited the original response (see references [15-19]).

At the level of an individual, sociality interacts with physiology to adapt the body to changing environmental conditions via concerted changes involving all of the anatomical systems. These adaptive physiological changes, which serve to maintain homeostasis, are active and thus draw from the body's overall energy budget. The concept of allostasis refers to the full set of (inter)active, adaptive physiological responses to physical or psychic challenges that are directed toward maintaining or returning the body to homeostasis $[2,12]$. The 'allostatic load' then can be thought of as the demand that allostasis places on the overall energy budget, with an increased allostatic load entailing decreased energy available for other processes. Given that there are fundamental differences in how energy is budgeted in males and females and how that partitioning is regulated by sex-specific neuroendocrine systems, it is not surprising that allostasis has sex-specific effects $[2,13]$. Thus, for example, it was recently shown that in men adaptation to chronic stress blunts the autonomic/cardiovascular response to acute stress without affecting the neuroendocrine response, which was associated with increased adiposity, whereas the opposite was true in women (blunted neuroendocrine response but augmented autonomic/cardiovascular response) [14]. While important to keep in mind, sex-specific effects will not be considered further here, as what follows are abstractions and generalizations that pertain to both sexes.

At the level of cellular biochemistry and molecular genetics, adaptation to a stimulus is generally mediated by negative feedback and incoherent feedforward regulatory loops [15-17], which work to return a system's output to baseline following its initial elevation in response to a sustained (i.e., chronic) increase in stimulus amplitude (Figure 1). Negative feedback occurs when an activator of a response to a stimulus also activates its own inhibitor (Figure 1A). Feedforward regulation occurs when the activator also activates a second regulator of the response itself, which can be either another activator (coherent feedforward regulation) or an inhibitor/repressor (incoherent feedforward regulation; Figure 1B). Adaptive regulatory circuits involving negative feedback and/or incoherent feedforward regulation establish 'set points' that determine the sensitivity thresholds that control system dynamics, for example by affording fold-change detection [18,19]. Thus, a system that becomes adapted to a chronically sustained increase in stimulus strength will respond again only when the fold-change, rather than to the absolute increment, of subsequent increases stimulus strength are equivalent to that of the original (Figure 1C). In this way, the adapted system manifests a new set point that renders it less sensitive to the original stimulus.

One of the more insidious effects of chronic stress and adaptation thereto is that it perturbs development of systems and/or life history stages that are developmentally plastic [20,21]. Developmental plasticity refers to the degree to which the phenotypic outcome of development is influenced by environmental conditions. In general, developmental plasticity is higher during early development than in later development. Physiological 
adaptation to chronic stress during a plastic stage of development can produce phenotypes that become locked in as plasticity is lost with subsequent developmental maturation, with lifelong or even intergenerational effects. Such 'developmental programming' is thought to be adaptive to the extent that it proactively prepares the organism for a stressful environment. However, it comes at a cost when the adaptations entail increased allostasis, and hence a greater allostatic load [12]. Moreover, adaptive developmental programming can be unhealthy when there is a mismatch between the anticipated conditions toward which the adaptations are directed and the actual conditions encountered by the mature organism, as the pre-adaptations can themselves be toxic under such conditions (e.g., as when nutritional stress directs development of a highly efficient metabolism that subsequently encounters a high calorie diet) [22].

The biomedical significance of stress-induced developmental programming is underscored by extensive epidemiology showing that early life adversity (ELA), in the form of either chronic stress (including prenatal exposure to chronic maternal stress) or intense acute stress (e.g., trauma) increases later life disease risk and susceptibility to neurological/psychological disturbances [23-26]. A common characteristic of many health problems that have developmental origins is chronic inflammation, affecting various tissues including the central nervous system (CNS) [26-28]. All in all, the data from epidemiology as well as experimental studies in model organisms indicates that stress-induced developmental programming contributes significantly to metabolic disease, dysregulation of the immune system and mental illness. Since the neuroendocrine stress system is inextricably linked to and a crucial regulator of metabolism, the immune system and mental health, it is reasonable to focus on the question of how its development is perturbed by stress, and chronic stress in particular from the standpoint of this review.

\section{The hypothalamus-pituitary-adrenal axis \& neuroendocrine regulation of homeostasis}

The two branches of the vertebrate neuroendocrine stress system are the sympathetic nervous system and the hypothalamus-pituitary-adrenal (HPA) axis. Both branches emanate from the hypothalamus and converge on the adrenal gland, working together to maintain homeostasis by orchestrating systemic physiological adaptations to environmental contingencies. The sympathetic nervous system provides the more immediate response to stress by promoting rapid release of epinephrine and norepinephrine from the adrenal medulla. The HPA axis, the main focus of this review, provides a more prolonged response critical for mitigating, resolving and adapting to the stress [29]. While the focus here is on the HPA axis and the glucocorticoid signaling pathway that it regulates, it is important to keep in mind that these are but part of (and thus dependent on) a larger system and physiological network, within which metabolic, autonomic and immune systems provide additional important mediators of adaptation [30].

The HPA axis is activated by circadian and stress-induced neurological signals that direct the periventricular nucleus (PVN) of the hypothalamus to produce corticotropic releasing hormone $(\mathrm{CRH})$, which binds specific receptors in the pituitary to induce production and secretion into the bloodstream of adrenocorticotropic hormone (ACTH). Receipt of circulating ACTH in the adrenal cortex stimulates production and secretion of glucocorticoids (GCs, primarily cortisol in humans), steroids that induce tissue-specific genomic responses throughout the body by binding the ubiquitously expressed glucocorticoid receptor (GR) as well as the more tissue-restricted mineralocorticoid receptor (MR). The GR and MR both function as sequence-specific DNA-binding transcription factors as well as non-nuclear signaling proteins. The GR binds GCs less avidly than the MR and is thus more dynamically regulated over the normal range of endogenous GC fluctuations.

GC-responsive gene expression maintains homeostasis both reactively, in response to unpredictable environmental stressors such as injury, trauma and starvation, and proactively, in anticipation of predictable environmental changes such as circadian activity cycles [29,31-34]. To that end, the HPA axis is highly dynamic, producing ultradian, circadian and stress-induced pulses of GCs that in turn regulate CRH and ACTH production via GR-mediated negative feedback on the hypothalamus and pituitary [35,36]. These dynamics are a functionally important aspect of GC signaling [37-41]. The ultradian pulsatility controls GR dwell time on its target sites in chromatin, which is important for responsivity of GR-mediated gene expression to changing conditions [37,41]. The circadian rhythm in GR activity helps entrain peripheral clocks to the central clock [32,42]. Circadian rhythms regulate much of physiology [43,44], including hematopoiesis, the immune system [42,45-48] and learning-dependent formation of synapses [49], whereas circadian dysregulation increases disease risk [48,50].

A major function of GC signaling is to regulate metabolism, as reflected in the name 'glucocorticoid' - the 'gluco' referring to glucose. Increased GC signaling, such as occurs with acute stress or at the beginning of the circadian activity phase (morning in diurnal species), leads to increased catabolism, for example, gluconeogenesis in the liver 
and lipolysis in adipocytes, increasing the availability of glucose and other energy substrates that feed increased mitochondrial respiration and energy production. Mitochondria represent a fascinating nexus for understanding GC signaling, as they are both the source and an ultimate target organelle for endogenous GCs such as cortisol [51]. The adrenal cortex, the major source of cortisol, can thus be viewed as a vertebrate organ specialized for coordinating nuclear genomic and mitochondrial activity throughout the body [51]. It is thus a key organ for regulating the flow of both information and energy in the service of homeostasis, arguably the defining characteristics of life itself.

Another major function of GC signaling is to regulate the immune system [52]. Colloquially, GCs are known for their immunosuppressant and anti-inflammatory activities, and synthetic GCs are widely prescribed to control inflammation. However, GC function with respect to the immune system is actually more complex, contributing to both activation and resolution of immune responses in ways that depend on concentration and/or temporal dynamics that can be construed as hormetic [53]. Thus, at low levels, GCs work to 'prime' immune cells and promote expression of genes involved in inflammation and the immune response, whereas higher levels have the opposite effects [53]. GCs regulate the immune system at least in part through their regulation of metabolism. An interesting case in point is GR-mediated regulation of the gene irgl, a target of the GR that encodes a mitochondrial enzyme expressed in macrophages [54]. The product of $\mathrm{irg} 1$ is an enzyme involved in fatty acid oxidation, a byproduct of which is reactive oxygen species (ROS) used to kill pathogens [54]. The hypothesis that GC-mediated regulation of the immune system is hormetic has an interesting implication: all else being equal, adaptation to increased levels of GC signaling via increased GC resistance in immunocytes would be expected shift the balance of stress-induced GC function toward the proinflammatory role normally associated with low GC levels.

A third major function of GC signaling is to regulate CNS activity. Although the GR has been the major focus of research on stress-induced glucocorticoid signaling throughout the body, the MR has emerged as an important player within the CNS, particularly within hippocampus where it mediates excitatory signaling in response to stress [55]. This has led to the MR:GR balance hypothesis [56] that the stress response is regulated by a dynamically shifting balance of activities of both the MR and GR that correlates with the different affinities of the two receptors for GCs. Thus early in the stress response as GC levels begin to rise, the higher affinity MR becomes engaged, activating rapid nongenomic excitatory responses that trigger emotion and memory retrieval within the CNS and contribute to the stress signaling through the HPA axis [56]. With higher GC levels, the lower affinity GR becomes engaged, orchestrating longer-term genomic responses directed toward providing energy, mitigating damage, storing memory [56] and finally terminating the response via negative feedback control of the HPA axis. Thus, according to the balance hypothesis, the MR and GR function antagonistically to the extent that the former is involved in mounting and the former in resolving the response to stress.

\section{GC signaling is dynamically \& adaptively regulated}

The hypothesis that the neuroendocrine stress system exerts its effects through a dynamically shifting balance of MR and GR activities fits well with the fact that endogenous GC levels are themselves highly dynamic, both under basal conditions and in response to acute stress. As noted above, under normal (unstressed) conditions, GCs undergo both ultradian and circadian fluctuations. The affinity of the GR for cortisol is such that the GR is only activated at peak levels of ultradian pulses, which therefore result in pulsatility of GR activation and engagement with the genome [37,41]. This serves to maximize the dynamic responsivity of the system. The ultradian rhythms are driven by a combined feedforward/feedback loop between the pituitary and adrenal cortex that produces phase-shifted oscillations of ACTH and cortisol $[57,58]$. The circadian rhythms in GC levels are driven by the central clock, and serve to coordinate metabolism with circadian activity cycles, and also to maintain synchrony between the autonomous circadian clocks of peripheral tissues with the central clock $[34,43,50,59]$. Moreover, the circadian rhythm has been shown to be required for efficient termination and hence transience of the HPA axis response to acute stress via GR-mediated negative feedback in the hypothalamus and pituitary [60,61].

Negative feedback regulation of neuroendocrine stress signaling mediates adaptation as well as dynamics. One of the genes activated by the GR is $f k b p 5$, which encodes the co-chaperone protein that inhibits activation GR by antagonizing binding of GCs thereto. Fkbp5 is thus an intracellular negative feedback regulator that increases resistance to the GC signal, adapting the system to chronically elevated GC levels as for example occurs in squirrel monkeys [62]. Other genes activated by the GR encode repressors of GR targets, affording incoherent feedforward regulation. Such regulators include the krüppel-like factors $k l f 9$, recently shown to function as a feedforward repressor of the GR target klf 2 in mouse macrophages [63], and klf15, a feedforward repressor of $m t 2 a$ and tiparp in mouse lungs [64]. Incoherent feedforward regulation enforces a pulsed response, even when the stimulus remains 
elevated, and in that way affords adaptation as discussed above (Figure 1). Thus feedback and feedforward loops work at multiple levels from the cellular to the systemic work to dynamically and adaptively control HPA axis output and signaling effects.

Among feedforward regulators of GC/GR signaling, Klf9 is emerging as a particularly interesting transcription factor for a number of reasons. First, it has been shown to collaborate with other transcription factors and neuroendocrine systems, including the thyroid receptor, progesterone receptor and Nrf2 [65-68]. Interestingly, with the latter it works as an incoherent feedforward regulator of the response to oxidative stress, amplifying ROS levels by repressing the expression of antioxidant enzymes [67]. In human epidermis, KLF9 functions as a cortisol-induced circadian regulator of keratinocyte proliferation [69]. In a recent meta-analysis of microarray studies of GC-induced gene expression in the brain or cells derived from the CNS of mice, rats, or humans, $k$ lf 9 was shown to be the most commonly upregulated transcriptional regulatory gene [70]. Finally, it was recently shown that Klf9 is responsible for chronic stress-induced remodeling of neural circuits in the mouse hippocampus leading to pathological fearconditioning and behavior [71], and for increased liver gluconeogenesis contributing to development of diabetes in response to chronic dexamethasone exposure [72]. Thus Klf9 appears to be an important feedforward transcriptional regulator of GC signaling that integrates the neuroendocrine stress system with other neuroendocrine and metabolic signaling systems.

\section{Chronic stress, cortisol dynamics \& HPA axis dysregulation}

From the foregoing, it should be apparent that one of the problems with chronic stress is that it produces a situation in which continuous stress-induced activation of the HPA axis occurs coincidentally and thus cross-purposes with negative feedback and incoherent feedforward regulation of its signaling activity. As a result, the dynamic regulation of the system is compromised, and cortisol levels become chronically elevated. While this is countered by adaptive mechanisms such as $f k b p 5$ activation that increase GC resistance, increased GC resistance can lead to chronic inflammation, a common feature of diseases associated with chronic stress [73]. The toxicity of chronic stress is thus likely to extend in part from the fact that the same feedback and feedforward circuits that normally mediate optimally dynamic functionality also mediate adaptation to chronically high GC levels, with the latter functionality compromising the former by establishing new set points affecting dynamic control (Figure 1). Loss of optimal dynamics due to adaptation to chronically high GC levels would be expected to have ramifying effects that increase allostatic load and reduce the adaptive capacity of the system. GCs are systemic signals affecting all of the body's tissues and organ systems [33], and therefore adaptive capacity is coordinately determined by regulatory systems engaged throughout the organism, any one of which can become limiting when diseased. Moreover, information flow along the brain-body axis is bidirectional, with the health of one affecting the health of the other (which points up the artificiality and danger of treating them as separate entities). Nevertheless, the CNS (and the brain in particular) is by definition central to this regulation, and its adaptive capacity is determined by its plasticity.

The CNS is a complex adaptive system that retains some amount of developmental plasticity throughout the life course. The plasticity manifests as experience-induced remodeling of neural architecture, notably within the hippocampus, wherein synaptic plasticity and neurogenesis are stimulated by acute stress but negatively impacted by chronic stress [74,75]. A major factor contributing to adaptive (or maladaptive) remodeling in response to chronic stress is excitatory neurotransmission mediated by glutamate, in part stimulated by glucocorticoids via nongenomic activities of the MR and GR [75,76]. Chronically elevated excitatory signaling can eventually lead to neurotoxicity and cell death associated with intracellular calcium overload. This is countered by activation of neuronal immediate early genes, in particular that encoding the transcription factor Npas4, which directs neuroprotective gene expression and development of inhibitory synapses [77-79]. Even so, stress-induced remodeling of neural circuitry within limbic regions of the CNS, in particular the hippocampus, will affect HPA axis regulation to the extent that the remodeled circuits impinge on the PVN and affect CRH production. One way that it might lead to dysregulation is by increasing CRH tone, which controls the ultradian pulsatility of ACTH and GCs, with higher CRH tone leading to decreased pulsatility (flatter GC profile) [58]. This would have adverse health consequences given the importance of GC pulsatility as discussed above. An implication of this line of reasoning is that optimal regulation of the HPA axis depends on an optimal excitatory/inhibitory balance within the hippocampus and other regions of the brain that signal to the PVN.

Plasticity within the CNS and peripheral tissues is subject to epigenetic regulation by chromatin modifications and micro-RNA expression. One well-studied epigenetic modification affected by chronic stress is DNA methylation. Methylation of cis-regulatory regions of genes (promoters and enhancers) can affect transcription factor binding, 
altering the responsivity of the gene to signals. A well-studied example in humans is FKBP5, a variant of which contains an intronic GR-binding site subject to stress-induced demethylation [80,81]. In individuals carrying this variant, the binding site is hypomethylated in response to childhood trauma, which allows increased accessibility to the GR and hence greater FKBP5 responsivity to GCs [80,81]. As a result, individuals with this variant overexpress FKBP5 in response to chronic stress, increasing GC resistance and risk of associated pathologies [82]. Other genes involved in GC signaling, including $n r 3 c 1$ which encodes the GR, are also subject to stress-induced epigenetic modification that affects their activity [83-86] and hence responsivity to GCs. Epigenetically programmed GC resistance within peripheral tissues or within the HPA axis itself, particularly within the pituitary affecting negative feedback regulation that is critical for dynamic control of GC signaling, can thus lead to dysregulation of the neuroendocrine stress system.

On the other hand, plasticity within the CNS also affords opportunities for interventions designed to counteract the adverse effects of chronic stress. For example, in mice exposed to 21 days of chronic restraint stress, the hippocampus was shown to have an adaptive response manifesting as resilience to a familiar stressor (acute restraint stress), which opened a transient 'window of plasticity' within which the negative behavioral and molecular effects of the chronic restraint stress were reversed [74]. This occurred through upregulation of the histone acetyl-transferase P300, leading to enhanced expression of mGlu2 and consequent inhibition of glutamate release [74]. This suggests that pharmacological interventions such as the acetyl donor acetyl-L-carnitine applied within defined windows of plasticity might be used to counteract some of the negative neurological effects of chronic stress, particularly those associated with glutamatergic excitotoxicity [74,87].

\section{Chronic early life stress \& developmental programming}

Developmental plasticity is generally maximal during immature stages of development, so that is when the neuroendocrine stress system might be expected to be most susceptible to chronic stress, and also more amenable to therapeutic interventions designed to counteract the negative effects of earlier insults. Indeed, while prenatal brain development is especially sensitive to perturbation by environmental factors, including elevated cortisol levels associated with chronic maternal stress, these effects can be counteracted to an extent by appropriate postnatal interventions such as high quality parent-infant interaction and bonding [88,89]. As noted above, human epidemiology and experimental studies with model organisms clearly indicate a correlation between ELA and HPA axis dysregulation and associated health problems later in life. The problems are both physical (e.g., metabolic syndrome, inflammatory diseases such as asthma) and mental (e.g., anxiety and depression) and typically associated with chronic inflammation indicating immune system dysregulation $[27,28,90-92]$. The latter is likely at least in part a secondary consequence of HPA axis dysregulation, given the critical role of GC signaling both in direct immunoregulation and circadian regulation, the latter also being necessary for optimal immune system function $[42,46,48,93]$. But while the correlation is well established and some of the mechanisms have been characterized, the gene regulatory networks through which early life stress and adversity affect development of the neuroendocrine stress system remain largely unknown.

One way that chronic stress is likely to affect development of the neuroendocrine stress system is through altered activities of the GR and MR, both of which are present at high levels in the developing brain. Their chronic activation may perturb development of stress-sensitive neural circuitry through their direct or indirect regulation of key plasticity genes (e.g., altered expression of klf9 or npas 4 ), and also via epigenetic effects such as altered DNA methylation (as discussed above with FKBP5), with long term effects on HPA axis regulation. Consistent with this scenario, a recent study found that children who had experienced ELA had smaller hippocampal volume and a flatter diurnal cortisol profile than controls [94]. Zebrafish embryos injected with cortisol show an altered pattern of neurogenesis in the fish equivalent of the hippocampus, and develop an aberrant behavioral phenotype [95]. The extent to which these phenotypes result from altered GR and MR activity/balance in response to chronically elevated GCs is not known and calls for more research.

\section{Conclusion}

The toxicity of chronic stress is likely to stem in large part from its chronicity, which overrides the highly dynamic regulation that is critical to optimal HPA axis function. Adaptation to chronically elevated GC levels leads to loss of system sensitivity and responsivity through chronic activation of negative feedback and feedforward regulators. When this occurs during early development, it can affect neuro-architecture in brain regions that regulate the HPA axis, leading to its long-term dysregulation. The GR and MR are GC-activated transcription factors and signaling 
proteins that normally regulate the stress system via a dynamically shifting balance controlled by the dynamic fluctuations of endogenous GCs and are hence prime molecular candidates for mediating the developmental programming effects of chronic stress and chronically elevated GC levels.

\section{Future perspective}

The genome directs development through gene regulatory networks defined by sequence-specific interactions between transcription factors and their DNA-binding sites [96,97], and the environment perturbs development by perturbing the operation of those networks in developmentally plastic systems. The gene regulatory networks downstream of the GR and MR that direct development of the neuroendocrine stress system are still largely uncharted. Future work in model systems subjected to stress or GC treatment, combined with high throughput sequencing approaches such as RNA-seq and ChIP-seq, will be required to comprehensively identify the relevant regulatory genes. Several candidates have been gleaned from the literature and work ongoing in our laboratory, including transcriptional regulatory genes such as klf9 and npas 4 as discussed above. Beyond identification of the genes themselves the linkages between them will need to be defined, as will their roles in regulating each others' expression dynamics, through judiciously designed perturbation (e.g., knockout, knockdown and pharmacological inhibition) studies that systematically assess the effects of the perturbation on all genes in the network, similar to what was done over the past 20 years to elucidate the developmental gene regulatory networks that control sea urchin embryogenesis [98-100]. Only in this way will the understanding of 'developmental programming' move from descriptive phenomenology to predictive molecular-genetic mechanism.

\section{Executive summary}

- Chronic stress undermines health by perturbing dynamic regulation of the neuroendocrine stress system via physiological adaptation and developmental programming.

- Physiological adaptation to stress and its sequelae entails allostasis, defined as the array of active adaptive processes needed for homeostasis.

- Allostasis draws on the overall energy budget, exacting an 'allostatic load' with sex-specific effects.

- Physiological adaptation during early development can have long term effects on allostasis and the allostatic load.

- The hypothalamus-pituitary-adrenal (HPA) axis contributes to allostasis as a critical neuroendocrine regulator of homeostasis.

- The HPA axis regulates systemic levels of GCs (e.g., cortisol) that activate the glucocorticoid receptor (GR) and mineralocorticoid receptor (MR), transcription factors and signaling proteins with different GC affinities.

- GCs regulate metabolism, the immune system and the CNS.

- The GR:MR balance hypothesis proposes that fluctuations of GCs dynamically control GC signaling and the stress response by way of a temporally shifting balance between MR and GR activities, the former working to mount the response and the latter to resolve it.

- The HPA axis regulates GC levels dynamically and adaptively.

- Endogenous GC levels display ultradian and circadian rhythms and transient responses to stress.

- Cortisol pulsatility is an important determinant of GR activity dynamics and regulation.

- Feedback and feedforward genetic regulators of GC signaling have been identified that mediate adaptation.

- Chronic stress alters GC signaling dynamics by way of HPA axis dysregulation.

- Chronic stress leads to constitutively elevated cortisol, overriding healthy dynamics and promoting development of GC resistance via adaptation.

- As a result, chronic stress promotes chronic inflammation.

- The stress system adapts to chronically elevated cortisol by way CNS plasticity and epigenetic effects on genes that control sensitivity to GCs.

- Chronic early life stress results in developmental programming that affects regulation of the neuroendocrine stress system.

- Early life stress is correlated with an increase in adult disease risk.

- High levels of GR and MR in the CNS are likely to affect its development in response to chronically elevated GCS associated with chronic stress, leading to altered CNS architecture upstream of the HPA axis and hence dysregulation of the latter.

- Conclusion and future perspective: understanding the mechanism underlying developmental programming of the neuroendocrine stress system will require research to define stress-responsive developmental gene regulatory networks engaged by the GR and MR. 
Author contributions

JA Coffman conceived and wrote the manuscript and drafted the figure.

Acknowledgments

The author thanks B McEwen for providing helpful comments on a presubmission draft of this article.

\section{Financial and competing interest disclosure}

The author's work is funded by the National Institutes of Health (GM103423, GM104318 and HD099468) and a Morris Scientific Discovery Award. The author has no other relevant affiliations or financial involvement with any organization or entity with a financial interest in or financial conflict with the subject matter or materials discussed in the manuscript apart from those disclosed.

No writing assistance was utilized in the production of this manuscript.

\section{Open access}

This work is licensed under the Attribution-NonCommercial-NoDerivatives 4.0 Unported License. To view a copy of this license, visit http://creativecommons.org/licenses/by-nc-nd/4.0/

\section{References}

Papers of special note have been highlighted as: $\bullet$ of interest; $\bullet \bullet$ of considerable interest

1. Doom JR, Gunnar MR. Stress physiology and developmental psychopathology: past, present, and future. Dev. Psychopathol. 25(4 Pt 2), 1359-1373 (2013).

2. McEwen BS. Redefining neuroendocrinology: epigenetics of brain-body communication over the life course. Front. Neuroendocrinol. 49 , $8-30$ (2017).

3. Halfon N, Larson K, Lu M, Tullis E, Russ S. Lifecourse health development: past, present and future. Matern. Child Health J. 18(2), 344-365 (2014).

4. Darwin C. On the Origin of Species by Means of Natural Selection. John Murray, London, UK (1859).

5. Shaw JR, Hampton TH, King BL et al. Natural selection canalizes expression variation of environmentally induced plasticity-enabling genes. Mol. Biol. Evol. 31(11), 3002-3015 (2014).

6. D’Urso A, Brickner JH. Mechanisms of epigenetic memory. Trends Genet. 30(6), 230-236 (2014).

7. Gilbert SF, Bosch TC, Ledon-Rettig C. Eco-Evo-Devo: developmental symbiosis and developmental plasticity as evolutionary agents. Nat. Rev. Genet. 16(10), 611-622 (2015).

8. Ganster DC, Victor B. The impact of social support on mental and physical health. Br. J. Med. Psychol. 61(Pt 1), 17-36 (1988).

9. Olstad R, Sexton H, Sogaard AJ. The Finnmark study. A prospective population study of the social support buffer hypothesis, specific stressors and mental distress. Soc. Psychiatry Psychiatr. Epidemiol. 36(12), 582-589 (2001).

10. Berger M, Sarnyai Z. "More than skin deep": stress neurobiology and mental health consequences of racial discrimination. Stress $18(1)$, $1-10(2015)$.

11. Masis-Calvo M, Schmidtner AK, de Moura Oliveira VE, Grossmann CP, de Jong TR, Neumann ID. Animal models of social stress: the dark side of social interactions. Stress 21(5), 417-432 (2018).

12. Danese A, McEwen BS. Adverse childhood experiences, allostasis, allostatic load, and age-related disease. Physiol. Behav. 106(1), 29-39 (2012).

13. McEwen BS, Milner TA. Understanding the broad influence of sex hormones and sex differences in the brain. J. Neurosci. Res. 95(1-2), 24-39 (2016).

14. Jones A, Pruessner JC, McMillan MR et al. Physiological adaptations to chronic stress in healthy humans - why might the sexes have evolved different energy utilisation strategies? J. Physiol. 594(15), 4297-4307 (2016).

15. Ma W, Trusina A, El-Samad H, Lim WA, Tang C. Defining network topologies that can achieve biochemical adaptation. Cell 138(4), 760-773 (2009).

- Dynamic modeling of idealized three-node biochemical networks and computer simulations covering parameter space were used to comprehensively search for network topologies that mediate adaptation, showing that only the only two sufficient for robust adaptation were negative feedback and incoherent feedforward regulation.

16. Ferrell JE Jr. Perfect and near-perfect adaptation in cell signaling. Cell Syst. 2(2), 62-67 (2016).

17. Shi W, Ma W, Xiong L, Zhang M, Tang C. Adaptation with transcriptional regulation. Sci Rep. 7, 42648 (2017).

18. Goentoro L, Shoval O, Kirschner MW, Alon U. The incoherent feedforward loop can provide fold-change detection in gene regulation. Mol. Cell 36(5), 894-899 (2009).

19. Adler M, Szekely P, Mayo A, Alon U. Optimal regulatory circuit topologies for fold-change detection. Cell Syst. 4(2), 171-181 (2017). 
20. Provencal N, Binder EB. The effects of early life stress on the epigenome: from the womb to adulthood and even before. Exp. Neurol. 268, 10-20 (2015).

21. Zannas AS, Chrousos GP. Epigenetic programming by stress and glucocorticoids along the human lifespan. Mol. Psychiatry 22(5), 640-646 (2017).

22. Lakshmy R. Metabolic syndrome: role of maternal undernutrition and fetal programming. Rev. Endocr. Metab. Disord. 14(3), 229-240 (2013).

23. Harris A, Seckl J. Glucocorticoids, prenatal stress and the programming of disease. Horm. Behav. 59(3), 279-289 (2011).

24. Khulan B, Drake AJ. Glucocorticoids as mediators of developmental programming effects. Best Pract. Res. Clin. Endocrinol. Metab. 26(5), 689-700 (2012).

25. Kalmakis KA, Chandler GE. Health consequences of adverse childhood experiences: a systematic review. J. Am. Assoc. Nurse Pract. 27(8), 457-465 (2015).

26. Berens AE, Jensen SKG, Nelson CA 3rd. Biological embedding of childhood adversity: from physiological mechanisms to clinical implications. BMC Med. 15(1), 135 (2017).

27. Chen T, Liu HX, Yan HY, Wu DM, Ping J. Developmental origins of inflammatory and immune diseases. Mol. Hum. Reprod. 22(8), 858-865 (2016).

28. Finnell JE, Wood SK. Putative inflammatory sensitive mechanisms underlying risk or resilience to social stress. Front. Behav. Neurosci. 12, 240 (2018).

29. Sapolsky RM, Romero LM, Munck AU. How do glucocorticoids influence stress responses? Integrating permissive, suppressive, stimulatory, and preparative actions. Endocr. Rev. 21(1), 55-89 (2000).

30. McEwen BS. What is the confusion with cortisol? Chronic Stress 3(1), 1-3 (2019).

31. Herman JP, McKlveen JM, Ghosal S et al. Regulation of the hypothalamic-pituitary-adrenocortical stress response. Compr. Physiol. 6(2), 603-621 (2016).

32. Dickmeis T. Glucocorticoids and the circadian clock. J. Endocrinol. 200(1), 3-22 (2009).

33. Kadmiel M, Cidlowski JA. Glucocorticoid receptor signaling in health and disease. Trends Pharmacol. Sci. 34(9), 518-530 (2013).

34. Spencer RL, Chun LE, Hartsock MJ, Woodruff ER. Glucocorticoid hormones are both a major circadian signal and major stress signal: how this shared signal contributes to a dynamic relationship between the circadian and stress systems. Front. Neuroendocrinol. 49, 52-71 (2018).

35. Dickmeis T, Weger BD, Weger M. The circadian clock and glucocorticoids - interactions across many time scales. Mol. Cell. Endocrinol. 380(1-2), 2-15 (2013).

36. Keller-Wood M. Hypothalamic-pituitary - adrenal axis-feedback control. Compr. Physiol. 5(3), 1161-1182 (2015).

37. Stavreva DA, Wiench M, John S et al. Ultradian hormone stimulation induces glucocorticoid receptor-mediated pulses of gene transcription. Nat. Cell Biol. 11(9), 1093-1102 (2009).

- Ultradian corticosteroid pulses result in pulses of glucocorticoid receptor (GR) occupancy of GC response elements in nuclear chromatin and pulsed expression of primary transcripts from GR target genes, both in cultured cells and animal models. The pulsing of nuclear GR activity occurs via its cycling through the chaperone machinery, which functions to deactivate the GR between pulses.

38. Stavreva DA, Coulon A, Baek S et al. Dynamics of chromatin accessibility and long-range interactions in response to glucocorticoid pulsing. Genome Res. 25(6), 845-857 (2015).

-• The effects on chromatin accessibility and transcription of pulsed versus continuous GC treatment of cultured cells were compared. Using an elegant and innovative way of visualizing genome-wide time course data, the authors showed that continuous treatment produces persistent transcriptional effects not seen with the pulsed treatment, hypothesized to be due to opportunistic transcription factor binding within more persistently open chromatin.

39. Miranda TB, Morris SA, Hager GL. Complex genomic interactions in the dynamic regulation of transcription by the glucocorticoid receptor. Mol. Cell. Endocrinol. 380(1-2), 16-24 (2013).

40. George AA, Schiltz RL, Hager GL. Dynamic access of the glucocorticoid receptor to response elements in chromatin. Int. J. Biochem. Cell Biol. 41(1), 214-224 (2009).

41. Conway-Campbell BL, Pooley JR, Hager GL, Lightman SL. Molecular dynamics of ultradian glucocorticoid receptor action. Mol. Cell. Endocrinol. 348(2), 383-393 (2012).

42. Dumbell R, Matveeva O, Oster H. Circadian clocks, stress, and immunity. Front. Endocrinol. (Lausanne) 7, 37 (2016).

43. Weger BD, Weger M, Gorling B et al. Extensive regulation of diurnal transcription and metabolism by glucocorticoids. PLoS Genet. 12(12), 1006512 (2016).

44. Karatsoreos IN, Bhagat S, Bloss EB, Morrison JH, McEwen BS. Disruption of circadian clocks has ramifications for metabolism, brain, and behavior. Proc. Natl Acad. Sci. USA 108(4), 1657-1662 (2011). 
45. Mendez-Ferrer S, Chow A, Merad M, Frenette PS. Circadian rhythms influence hematopoietic stem cells. Curr. Opin. Hematol. 16(4), 235-242 (2009).

46. Keller M, Mazuch J, Abraham U et al. A circadian clock in macrophages controls inflammatory immune responses. Proc. Natl Acad. Sci. USA 106(50), 21407-21412 (2009).

47. Arjona A, Sarkar DK. Are circadian rhythms the code of hypothalamic-immune communication? Insights from natural killer cells. Neurochem. Res. 33(4), 708-718 (2008).

48. Man K, Loudon A, Chawla A. Immunity around the clock. Science 354(6315), 999-1003 (2016).

49. Liston C, Cichon JM, Jeanneteau F, Jia Z, Chao MV, Gan WB. Circadian glucocorticoid oscillations promote learning-dependent synapse formation and maintenance. Nat. Neurosci. 16(6), 698-705 (2013).

50. Oster $\mathrm{H}$, Challet $\mathrm{E}, \mathrm{Ott} \mathrm{V}$ et al. The functional and clinical significance of the 24-h rhythm of circulating glucocorticoids. Endocr. Rev. er20151080 (2016).

51. Picard M, McEwen BS, Epel ES, Sandi C. An energetic view of stress: focus on mitochondria. Front. Neuroendocrinol. 49, 72-85 (2018).

52. Dhabhar FS. Stress-induced augmentation of immune function-the role of stress hormones, leukocyte trafficking, and cytokines. Brain Behav. Immun. 16(6), 785-798 (2002).

53. Yeager MP, Pioli PA, Guyre PM. Cortisol exerts bi-phasic regulation of inflammation in humans. Dose Response 9(3), 332-347 (2011).

54. Hall CJ, Boyle RH, Astin JW et al. Immunoresponsive gene 1 augments bactericidal activity of macrophage-lineage cells by regulating beta-oxidation-dependent mitochondrial ROS production. Cell Metab. 18(2), 265-278 (2013).

55. Karst H, Berger S, Turiault M, Tronche F, Schutz G, Joels M. Mineralocorticoid receptors are indispensable for nongenomic modulation of hippocampal glutamate transmission by corticosterone. Proc. Natl Acad. Sci. USA 102(52), 19204-19207 (2005).

56. de Kloet ER. From receptor balance to rational glucocorticoid therapy. Endocrinology 155(8), 2754-2769 (2014).

57. Walker JJ, Terry JR, Lightman SL. Origin of ultradian pulsatility in the hypothalamic-pituitary-adrenal axis. Proc. Biol. Sci. 277(1688), 1627-1633 (2010).

58. Walker JJ, Spiga F, Waite E et al. The origin of glucocorticoid hormone oscillations. PLoS Biol. 10(6), e1001341 (2012).

-• In vivo experimental validation of a previously proposed computational model showing that the ultradian oscillations of endogenous GCs are produced entirely by coupled feedforward and feedback loops between adrenocorticotropic hormone produced by the pituitary and corticosteroid produced by the adrenal cortex. Pulsed corticotropic releasing hormone (CRH) from the hypothalamus is not required, but the ultradian GC pulsatility is critically dependent on CRH tone, being lost with chronically high CRH.

59. Cuesta M, Cermakian N, Boivin DB. Glucocorticoids entrain molecular clock components in human peripheral cells. FASEB J. 29(4), 1360-1370 (2015).

60. Akana SF, Jacobson L, Cascio CS, Shinsako J, Dallman MF. Constant corticosterone replacement normalizes basal adrenocorticotropin (ACTH) but permits sustained ACTH hypersecretion after stress in adrenalectomized rats. Endocrinology 122(4), 1337-1342 (1988).

61. Jacobson L, Akana SF, Cascio CS, Shinsako J, Dallman MF. Circadian variations in plasma corticosterone permit normal termination of adrenocorticotropin responses to stress. Endocrinology 122(4), 1343-1348 (1988).

62. Westberry JM, Sadosky PW, Hubler TR, Gross KL, Scammell JG. Glucocorticoid resistance in squirrel monkeys results from a combination of a transcriptionally incompetent glucocorticoid receptor and overexpression of the glucocorticoid receptor co-chaperone FKBP51. J. Steroid Biochem. Mol. Biol. 100(1-3), 34-41 (2006).

63. Chinenov Y, Coppo M, Gupte R, Sacta MA, Rogatsky I. Glucocorticoid receptor coordinates transcription factor-dominated regulatory network in macrophages. BMC Genomics 15, 656 (2014).

- Transcriptional profiling of resting and lipopolysaccharide-stimulated mouse macrophages revealed that the GR is a central hub in a gene regulatory network that also including numerous KLF factors, some of which functioned as feedforward regulators of GR signalling. Among the latter, Klf9 was shown to be a GR-activated feedforward repressor of klf2.

64. Sasse SK, Mailloux CM, Barczak AJ et al. The glucocorticoid receptor and KLF15 regulate gene expression dynamics and integrate signals through feed-forward circuitry. Mol. Cell. Biol. 33(11), 2104-2115 (2013).

65. Pabona JM, Simmen FA, Nikiforov MA et al. Kruppel-like factor 9 and progesterone receptor coregulation of decidualizing endometrial stromal cells: implications for the pathogenesis of endometriosis. J. Clin. Endocrinol. Metab. 97(3), E376-392 (2012).

66. Denver RJ, Williamson KE. Identification of a thyroid hormone response element in the mouse Kruppel-like factor 9 gene to explain its postnatal expression in the brain. Endocrinology 150(8), 3935-3943 (2009).

67. Zucker SN, Fink EE, Bagati A et al. Nrf2 amplifies oxidative stress via induction of Klf9. Mol Cell 53(6), 916-928 (2014).

68. Cvoro A, Devito L, Milton FA et al. A thyroid hormone receptor/KLF9 axis in human hepatocytes and pluripotent stem cells. Stem Cells 33(2), 416-428 (2015).

69. Sporl F, Korge S, Jurchott $\mathrm{K}$ et al. Kruppel-like factor 9 is a circadian transcription factor in human epidermis that controls proliferation of keratinocytes. Proc. Natl Acad. Sci. USA 109(27), 10903-10908 (2012). 
70. Juszczak GR, Stankiewicz AM. Glucocorticoids, genes and brain function. Prog. Neuropsychopharmacol. Biol. Psychiatry 82, 136-168 (2018).

71. Besnard A, Langberg T, Levinson S et al. Targeting Kruppel-like factor 9 in excitatory neurons protects against chronic stress-induced impairments in dendritic spines and fear responses. Cell Rep. 23(11), 3183-3196 (2018).

-. Showed that pathological changes in neuronal architecture and behavior induced in mice by chronic stress depend on Klf9, which is transiently upregulated following the onset of the stress. Furthermore, KLF9 expression was found to be elevated in hippocampuses of women with recent chronic stress and major depressive disorder.

72. Cui A, Fan H, Zhang Y et al. Dexamethasone-induced Kruppel-like factor 9 expression promotes hepatic gluconeogenesis and hyperglycemia. J. Clin. Invest. 130, 2266-2278 (2019).

73. Cohen S, Janicki-Deverts D, Doyle WJ et al. Chronic stress, glucocorticoid receptor resistance, inflammation, and disease risk. Proc. Natl Acad. Sci. USA 109(16), 5995-5999 (2012).

74. Nasca C, Zelli D, Bigio B et al. Stress dynamically regulates behavior and glutamatergic gene expression in hippocampus by opening a window of epigenetic plasticity. Proc. Natl Acad. Sci. USA 112(48), 14960-14965 (2015).

75. Popoli M, Yan Z, McEwen BS, Sanacora G. The stressed synapse: the impact of stress and glucocorticoids on glutamate transmission. Nat. Rev. Neurosci. 13(1), 22-37 (2011).

76. McEwen BS. Neurobiological and systemic effects of chronic stress. Chronic Stress (Thousand Oaks) 1, 1 (2017).

77. Bloodgood BL, Sharma N, Browne HA, Trepman AZ, Greenberg ME. The activity-dependent transcription factor NPAS4 regulates domain-specific inhibition. Nature 503(7474), 121-125 (2013).

78. Spiegel I, Mardinly AR, Gabel HW et al. Npas4 regulates excitatory-inhibitory balance within neural circuits through cell-type-specific gene programs. Cell 157(5), 1216-1229 (2014).

-• Showed that the neural activity dependent transcription factor Npas4 directs different gene expression programs in excitatory and inhibitory neurons, directing the former to express genes that attract inhibitory synapses, and the latter to express genes that attract excitatory synapses, thereby increasing overall inhibition across the neural circuit.

79. Woitecki AM, Muller JA, van Loo KM, Sowade RF, Becker AJ, Schoch S. Identification of Synaptotagmin 10 as effector of NPAS4-mediated protection from excitotoxic neurodegeneration. J. Neurosci. 36(9), 2561-2570 (2016).

80. Binder EB. The role of FKBP5, a co-chaperone of the glucocorticoid receptor in the pathogenesis and therapy of affective and anxiety disorders. Psychoneuroendocrinology 34(Suppl. 1), S186-S195 (2009).

81. Klengel T, Mehta D, Anacker C et al. Allele-specific FKBP5 DNA demethylation mediates gene-childhood trauma interactions. Nat. Neurosci. 16(1), 33-41 (2012).

-• Showed that FKBP5 sequence variants associated with increased disease risk following childhood trauma contain an intronic GR-binding site that preferentially interacts with the transcriptional start site, and as a result is demethylated in response to the trauma, leading to higher levels of FKBP5 expression and consequent GC resistance. This occurs both in immune cells and brain regions associated with the stress system.

82. Zannas AS, Wiechmann T, Gassen NC, Binder EB. Gene-stress-epigenetic regulation of FKBP5: clinical and translational implications. Neuropsychopharmacology 41(1), 261-274 (2016).

83. Tyrka AR, Ridout KK, Parade SH. Childhood adversity and epigenetic regulation of glucocorticoid signaling genes: associations in children and adults. Dev. Psychopathol. 28(4pt2), 1319-1331 (2016).

84. Tyrka AR, Price LH, Marsit C, Walters OC, Carpenter LL. Childhood adversity and epigenetic modulation of the leukocyte glucocorticoid receptor: preliminary findings in healthy adults. PLoS ONE 7(1), e30148 (2012).

85. Bockmuhl Y, Patchev AV, Madejska A et al. Methylation at the $\mathrm{CpG}$ island shore region upregulates Nr3c1 promoter activity after early-life stress. Epigenetics 10(3), 247-257 (2015).

86. Liu PZ, Nusslock R. How stress gets under the skin: early life adversity and glucocorticoid receptor epigenetic regulation. Curr. Genomics 19(8), 653-664 (2018).

87. Nasca $\mathrm{C}, \mathrm{Xenos} \mathrm{D}$, Barone $\mathrm{Y}$ et al. L-acetylcarnitine causes rapid antidepressant effects through the epigenetic induction of $\mathrm{mGlu2}$ receptors. Proc. Natl Acad. Sci. USA 110(12), 4804-4809 (2013).

88. Buss C, Entringer S, Swanson JM, Wadhwa PD. The role of stress in brain development: the gestational environment's long-term effects on the brain. Cerebrum 2012, 4 (2012).

89. Dubovicky M. Neurobehavioral manifestations of developmental impairment of the brain. Interdiscip. Toxicol. 3(2), 59-67 (2010).

90. Miller GE, Chen E, Fok AK et al. Low early-life social class leaves a biological residue manifested by decreased glucocorticoid and increased proinflammatory signaling. Proc. Natl Acad. Sci. USA 106(34), 14716-14721 (2009).

91. Hartig EI, Zhu S, King BL, Coffman JA. Cortisol-treated zebrafish embryos develop into pro-inflammatory adults with aberrant immune gene regulation. Biol. Open 5(8), 1134-1141 (2016).

92. Mesquita AR, Wegerich Y, Patchev AV et al. Glucocorticoids and neuro- and behavioural development. Semin. Fetal. Neonatal. Med. 14(3), 130-135 (2009). 
93. Curtis AM, Bellet MM, Sassone-Corsi P, O’Neill LA. Circadian clock proteins and immunity. Immunity 40(2), 178-186 (2014).

94. Dahmen B, Puetz VB, Scharke W, von Polier GG, Herpertz-Dahlmann B, Konrad K. Effects of early-life adversity on hippocampal structures and associated hpa axis functions. Dev. Neurosci. 40(1), 13-22 (2018).

95. Best C, Kurrasch DM, Vijayan MM. Maternal cortisol stimulates neurogenesis and affects larval behaviour in zebrafish. Sci. Rep. 7, 40905 (2017).

96. Peter IS, Davidson EH. Genomic Control Process: Development and Evolution. 1st Edition Academic Press, Elsevier Inc. (2015).

97. Davidson EH. The Regulatory Genome: Gene Regulatory Networks in Development and Evolution. Academic Press/Elsevier, CA, USA (2006).

98. Peter IS, Davidson EH. A gene regulatory network controlling the embryonic specification of endoderm. Nature 474(7353), 635-639 (2011).

99. Su YH, Li E, Geiss GK, Longabaugh WJ, Kramer A, Davidson EH. A perturbation model of the gene regulatory network for oral and aboral ectoderm specification in the sea urchin embryo. Dev. Biol. 329(2), 410-421 (2009).

100. Ben-Tabou de-Leon S, Davidson EH. Experimentally based sea urchin gene regulatory network and the causal explanation of developmental phenomenology. Wiley Interdiscip. Rev. Syst. Biol. Med. 1(2), 237-246 (2009). 